Bangladesh J. Pl. Breed. Genet., 23(1): 01-07, 2010

\title{
HETEROSIS OF YIELD AND ITS CONTRIBUTING CHARACTERS OF T-AMAN RICE (Oryza sativa L)
}

\author{
P. C. Roy, M. G. Rasul, M. A. K. Mian and M. A. H. Molla ${ }^{1}$ \\ Department of Genetics and Plant Breeding \\ Bangabandhu Sheikh Mujibur Rahman Agricultural University \\ Gazipur 1706, Bangladesh
}

\begin{abstract}
The investigation was undertaken to study heterosis over better parent, heterosis over mid parent, reciprocal effects in eight crosses, their reciprocals and parents of T-Aman rice genotypes. Maximum traits showed heterosis in desirable direction of which the hybrid FR-13A $\times$ Arman sarder performed the best towards shorter stature, yield components like tiller number, panicle length, filled grains per panicle and 100-grain weight. The hybrid FR-13A $\times$ PJT (st) showed significant and positive heterosis over better parent for plant height, number of tillers per hill, grains per panicle and yield per hill which performed the best in terms of both physical as well as yield contributing traits. Considering heterosis over mid parent, the hybrid FR-13A $\times$ Arman Sarder performed the best showing heterosis in desirable direction on maximum number of traits namely tiller number, panicle length, filled grains per panicle, harvest index and yield per tiller. Most of the hybrids showed maternal effects but the cross FR-13A $\times$ PJT (st) had no maternal effects for 100 grains weight.
\end{abstract}

Key words: Rice, heterosis, better parent, mid parent, reciprocal effect

\section{INTRODUCTION}

Rice occupies about $75 \%$ of the total cropped area and constitutes $94 \%$ of cereals production. In Bangladesh total production area of rice is 64.63 lac ha and total

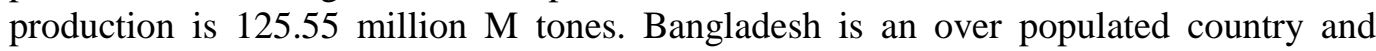
population is increasing day by day at rapid rate. At present, the rice growing areas are gradually decreased due to industrialization, housing and expansion of urban areas. The present population of 145 million will increase to 233 million by 2030 requiring 48 million tons of extra food grain (Karim et al. 1990). Under such conditions, all the additional production must come from higher yield due to non-availability of lands for expansion of rice. It is not possible to increase the production of rice horizontally due to lack of land. On the contrary, among the three types of rice Aus, Aman and Boro; Aman is fully dependent on natural condition. It is required for the rainy season but flood or submergence condition can hampered the expected production. Sometimes, rice plants may be damaged due to submergence for long time which affects the production of rice. So we should improve the production of rice vertically utilizing every resources and opportunity judiciously. Hybrid rice technology offers considerable opportunity for Bangladesh to increase the productivity of rice. Therefore, efficient and economic seed production package is one of the pre-requisites for the exploitation of heterosis on

\footnotetext{
${ }^{1}$ Department of Bioenvironmental Science, Bangabandhu Sheikh Mujibur Rahman Agricultural University, Gazipur 1706, Bangladesh.
} 
commercial scale. Considering these problems, an experiment was conducted to evaluate the yield related characteristics of nine parents and their $F_{1}$ generation obtained by crossing some submergence tolerant rice genotype.

\section{MATERIALS AND METHODS}

The experiment was carried out at the experimental farm of Bangabandhu Sheikh Mujibur Rahman Agricultural University, Salna, Gazipur during July 2007 to January 2009. The experiment was laid out in a Randomized Complete Block Design (RCBD) with three replications. Five rows of $4 \mathrm{~m}$ each constituted the experimental unit. Twenty five treatments were distributed in the experimental unit through randomization by using the IRRISTAT programme. Nine genotypes were used as parent material. Eight crosses and their reciprocals were made during first year (July 2007 to December 2007) of the experiment. In this case, the genotype FR-13A was used as both male and female for crossing with other genotypes. Then the experiment was conducted in the next year (July 2008 to January 2009) using eight crosses, their reciprocals and parents. Healthy seedlings of 30 days old were transplanted in separate strips of the experimental field. In each strip $20 \mathrm{~cm} \times 20 \mathrm{~cm}$ spacing between plant to plant and row to row, respectively were maintained. The overall mean value for each parent or hybrid in all the three replications for each character was taken for the estimation of heterosis. Heterosis was calculated as percent deviation of the $\mathrm{F}_{\mathrm{I}}$ hybrid from the mid parental value between two corresponding parents (Liang et al. 1971). The magnitude of heterosis was expressed as heterosis over better parent (BP) and heterosis over mid parent (MP) for 15 traits.

\section{RESULTS AND DISCUSSION}

\section{Heterosis over better parent}

Among the 16 hybrids, 10 had the significant negative heterosis for days to $50 \%$ flowering and days to maturity which is highly desirable (Table 1). Young and Virmani (1990) similarly observed negative mean heterosis, heterobeltiosis and standard heterosis for days to 50\% flowering. On the other hand, Patnaik et al. (1990) had not found any hybrid, earlier than the earliest parents. The heterosis of plant height ranged from 40.05 to $0.88 \%$. Among the 16 hybrids, 12 of them exhibited highly significant and positive heterosis over the better parent except FR-13A $\times$ Chamara. The mean heterosis over better parent was $34.34 \%$. Patnaik et al. (1990) did not find any hybrids, which was shorter than the shortest parent while studying different CMS lines. Young and Virmani (1990) mentioned that heterosis for plant height did not change the plant type of hybrids from semi-dwarf to tall because the parents possessed semi dwarfing gene. For number of tillers per hill all the hybrids showed significant positive heterosis effect at 5\% level except four hybrids. Lokaprakash et al. (1992) pointed out the importance of heterosis of productive tillers on heterosis of yield. For panicle length nine crosses showed significant effect at $1 \%$ level while three hybrids showed significant at $5 \%$ level and the rest were non-significant. Among the 16 hybrids, majority (9) of them exhibited positive heterosis over the better parent. Seven hybrids had the negative heterosis. Among the 16 , few hybrid exhibited negative heterosis over the better parent and only 5 hybrid, had the positive heterosis. Heterosis over better parent was significant $(1 \%)$ for the trait secondary branches per panicle. Among the 16 hybrids, only six exhibited positive heterosis over the better parent. Majority (9) of them had the negative heterosis. The cross FR-13A $\times$ PJM (st) showed positive non-significant heterosis. 
Table 1. Estimation of heterosis over better parent in 16 crosses in T. Aman rice

\begin{tabular}{|c|c|c|c|c|c|c|c|c|c|c|c|c|c|c|c|}
\hline Hybrids & $\begin{array}{l}\text { Days to first } \\
\text { flowering }\end{array}$ & $\begin{array}{c}\text { Days to } \\
50 \% \\
\text { flowering }\end{array}$ & $\begin{array}{c}\text { Days to } \\
100 \% \\
\text { flowering }\end{array}$ & $\begin{array}{l}\text { Days to } \\
\text { maturity }\end{array}$ & $\begin{array}{l}\text { Plant } \\
\text { height } \\
(\mathrm{cm})\end{array}$ & $\begin{array}{l}\text { No. of } \\
\text { tillers } \\
\text { per hill }\end{array}$ & $\begin{array}{l}\text { Panicle } \\
\text { length } \\
(\mathrm{cm})\end{array}$ & $\begin{array}{c}\text { Primary } \\
\text { branches } \\
\text { per } \\
\text { panicle } \\
\end{array}$ & $\begin{array}{l}\text { Secondary } \\
\text { branches } \\
\text { per panicle }\end{array}$ & $\begin{array}{l}\text { Yield per } \\
\text { hill }(\mathrm{g})\end{array}$ & $\begin{array}{l}\text { Yield } \\
\text { per } \\
\text { tiller(g) }\end{array}$ & $\begin{array}{l}\text { Harvest } \\
\text { index }\end{array}$ & $\begin{array}{l}\text { Filled } \\
\text { grain }\end{array}$ & $\begin{array}{l}\text { Spikelet } \\
\text { sterility }\end{array}$ & $\begin{array}{l}\text { Wt.of } \\
100 \\
\text { grain }(\mathrm{g})\end{array}$ \\
\hline FR-13A $\times$ BR-22 & $-3.58 \mathrm{~ns}$ & $-5.64 * *$ & $-13.4 \mathrm{~ns}$ & $-9.55^{* *}$ & $31.64 * *$ & $113.4^{*}$ & $12.2^{*}$ & $-5.05 \mathrm{~ns}$ & $33.61 * *$ & $-20.3 * *$ & $-64.7 * *$ & $-60.7 * *$ & $-24.2 * *$ & $351.0^{* * *}$ & $-10 \mathrm{~ns}$ \\
\hline FR-13A $\times$ BR-23 & $1.30 \mathrm{~ns}$ & $-0.31 \mathrm{~ns}$ & $1.465 \mathrm{~ns}$ & $-0.89 * *$ & $30.56^{* *}$ & $53.84 *$ & $2.28 \mathrm{~ns}$ & $5.59 \mathrm{~ns}$ & $23.41^{* *}$ & 105. ** & $46.8 * *$ & $-51.3 * *$ & $44.01 * *$ & $4.76 \mathrm{~ns}$ & $-23 . \mathrm{ns}$ \\
\hline $\mathrm{FR}-13 \mathrm{~A} \times \mathrm{PJM}(\mathrm{st})$ & $-1.30 \mathrm{~ns}$ & $-3.1 * *$ & $-6.15 \mathrm{~ns}$ & $-8.14 * *$ & $5.70 \mathrm{~ns}$ & $-6.02 \mathrm{~ns}$ & $-2.62 \mathrm{~ns}$ & $0.62 \mathrm{~ns}$ & $0.48 \mathrm{~ns}$ & $-4.4 * *$ & $-5.2 \mathrm{~ns}$ & $360.0 * *$ & $16.76^{* * *}$ & $10.57 \mathrm{~ns}$ & $15.6 \mathrm{~ns}$ \\
\hline FR-13A $\times$ PJT (st) & $-13.0 \mathrm{~ns}$ & $-10.6 * *$ & $-11.37 \mathrm{~ns}$ & $-9.68 \mathrm{~ns}$ & $38.62 * *$ & $139.0^{*}$ & $7.214^{*}$ & $6.67 \mathrm{~ns}$ & $-4.38 \mathrm{~ns}$ & 181. ** & $15.3 \mathrm{~ns}$ & $-64.06 * *$ & $-35.2 * *$ & $171.5^{* *}$ & $-20 \mathrm{~ns}$ \\
\hline FR-13A $\times$ Chamara & 0 & $-2.19 \mathrm{~ns}$ & $-4.39 \mathrm{~ns}$ & $-0.89 \mathrm{~ns}$ & -4.37 & $-35.5^{*}$ & $-0.57 * *$ & $-16.4 * *$ & $-8.09 \mathrm{~ns}$ & $-31.3 * *$ & $-5.4 \mathrm{~ns}$ & $29.41 \mathrm{~ns}$ & $-5.57 \mathrm{~ns}$ & $97.23 \mathrm{~ns}$ & $6.66 \mathrm{~ns}$ \\
\hline FR-13A $\times$ Heachua & $-3.06 \mathrm{~ns}$ & $1.306^{* *}$ & $2.57 \mathrm{~ns}$ & 0 & $13.47^{* *}$ & $103.1^{*}$ & $-4.42 * *$ & $-21.6^{* *}$ & $-3.94 \mathrm{~ns}$ & 109. ** & $-16.8 * *$ & $26.47 \mathrm{~ns}$ & $48.99 * *$ & $-25.10^{* *}$ & $30 \mathrm{~ns}$ \\
\hline FR-13A $\times$ Khaiyamota & $-9.12 \mathrm{~ns}$ & $-5.95 * *$ & $-10.52 \mathrm{~ns}$ & $-8.08 * *$ & $29.87 * *$ & $82.25^{*}$ & $4.93 * *$ & $-16^{* *}$ & $-6.72 \mathrm{~ns}$ & $97.3 * *$ & $-23.6 * *$ & $-47.3 * *$ & $-36.0^{* *}$ & $299.0 \mathrm{~ns}$ & $-10 \mathrm{~ns}$ \\
\hline FR-13A $\times$ Arman sarder & $-10.7 \mathrm{~ns}$ & $-10.0 * *$ & $-11.8 \mathrm{~ns}$ & $-12.38^{* * *}$ & $33.59 * *$ & $82.97 *$ & $5.46^{* *}$ & $11.8^{* *}$ & $40.05^{* *}$ & $164^{* *}$ & $83.9 * *$ & $90.2 * *$ & $68.3^{* *}$ & $-36.0 \mathrm{~ns}$ & $20 \mathrm{~ns}$ \\
\hline BR-22 $\times$ FR-13A & $-5.86 \mathrm{~ns}$ & $-5.9 * *$ & $-13.2 \mathrm{~ns}$ & $-6.37 * *$ & $29.82 * *$ & $103.1^{*}$ & $8.44 * *$ & $-2.24 * *$ & $38.6^{* *}$ & $-14.9^{* *}$ & $-64 . * *$ & $57.04 * *$ & $-39.0^{* *}$ & $299.3^{* *}$ & $-16 . \mathrm{ns}$ \\
\hline BR-23 $\times$ FR-13A & $0.65 \mathrm{~ns}$ & $-1.56 \mathrm{~ns}$ & $-5.42 \mathrm{~ns}$ & $-3.47^{* *}$ & $40.05^{* *}$ & $98.29 *$ & $14.22^{* * *}$ & $11.8 \mathrm{~ns}$ & $80.0^{* *}$ & $-43 . * *$ & $-77 . * *$ & $57.69 * *$ & $31.7^{* *}$ & $166.1^{* *}$ & $-51 . \mathrm{ns}$ \\
\hline $\operatorname{PJM}(\mathrm{st}) \times$ FR-13A & $0.977 \mathrm{~ns}$ & $-0.31 \mathrm{~ns}$ & $2.057 \mathrm{~ns}$ & $0.46 \mathrm{~ns}$ & $0.88 \mathrm{~ns}$ & $-10.3 \mathrm{~ns}$ & $-7.01 * *$ & $-10.4 * *$ & $-34.0^{* *}$ & $-32 . * *$ & $-17.1 * *$ & $-40.4^{* *}$ & $-19.6^{* *}$ & $4.17 \mathrm{~ns}$ & $9 \mathrm{~ns}$ \\
\hline PJT (st) $\times$ FR-13A & $-12 . \mathrm{ns}$ & $-11.3 * *$ & $-10.75 \mathrm{~ns}$ & $-10.22^{* * *}$ & $30.84 * *$ & $115.2^{*}$ & $-3.90 *$ & $-14 * *$ & $-14.3 * *$ & $114^{* * *}$ & $6.74 * *$ & $-76.5 * *$ & $-60.0^{* *}$ & $101.4^{*}$ & $-20 \mathrm{~ns}$ \\
\hline Chamara $\times$ FR-13A & $2.606 \mathrm{~ns}$ & $-8.14 * *$ & $-1.73 \mathrm{~ns}$ & $-0.44 \mathrm{~ns}$ & $5.37 \mathrm{~ns}$ & $55.03 *$ & $1.63 \mathrm{~ns}$ & $-18.8^{* *}$ & $-7.22 \mathrm{~ns}$ & $43.0 * *$ & $-12.8 \mathrm{~ns}$ & $2.94 \mathrm{~ns}$ & $-44.5^{* *}$ & $87.56 \mathrm{~ns}$ & $-10 \mathrm{~ns}$ \\
\hline Heachua $\times$ FR-13A & $4.4 \mathrm{~ns}$ & $1.96 \mathrm{~ns}$ & $2.56 \mathrm{~ns}$ & $8.91^{* *}$ & $17.34 * *$ & $57.44 *$ & $-6.16^{* *}$ & $-11.6^{* *}$ & $-6.89 \mathrm{~ns}$ & $43.7 * *$ & $-17.1^{*}$ & 0 & $6.70 \mathrm{~ns}$ & $-7.62 \mathrm{~ns}$ & $16.6 \mathrm{~ns}$ \\
\hline Khaiyamota $\times$ FR-13A & $-8.794 \mathrm{~ns}$ & $-9.40^{* *}$ & $-9.93 \mathrm{~ns}$ & $-9.74 * *$ & $17.46^{* *}$ & $57.01 *$ & $10.7^{* *}$ & $-9.33^{*}$ & $-17.2^{* *}$ & $49.3 * *$ & $-25.2 * *$ & $-39.5 * *$ & $-18.9^{* *}$ & $311.5 \mathrm{~ns}$ & $33.3 \mathrm{~ns}$ \\
\hline Arman sarder $\times$ FR-13A & $-4.88 \mathrm{~ns}$ & $-5.6 * *$ & $-9.67 \mathrm{~ns}$ & $-6.17 * *$ & $19.73^{* *}$ & $15.95 \mathrm{~ns}$ & $-1.43 \mathrm{~ns}$ & $-3.94 \mathrm{~ns}$ & $14.03^{* *}$ & $-31 . * *$ & $-25.0 * *$ & $45 \mathrm{~ns}$ & $-50.0^{* *}$ & $56.1 \mathrm{~ns}$ & $-10 \mathrm{~ns}$ \\
\hline & $\begin{array}{c}-6.24 \\
\end{array}$ & -7.72 & -10.9 & -7.63 & 34.34 & 64.06 & 2.56 & -5.82 & 7.96 & 45.6 & -12.6 & 43.0 & $\begin{array}{l}-7.31 \\
\end{array}$ & 189.1 & -2.4 \\
\hline & 5 & 2 & 4 & 2 & 15 & 13 & 9 & 5 & 7 & 9 & 4 & 8 & 6 & 13 & 7 \\
\hline No. of negative heterosis & 10 & 14 & 12 & 13 & 1 & 3 & 7 & 11 & 9 & 10 & 12 & 7 & 10 & 3 & 9 \\
\hline
\end{tabular}

*indicates significant at 5\% level and ** indicates significant at $1 \%$ level 


\section{Heterosis over mid parent}

Among the 16 hybrids, only one exhibited positive heterosis over the mid parent. Majority (15) of them had the negative heterosis. Majority of the hybrids showed significant effect at $1 \%$ level, only two hybrids showed non-significant effect. The range of the heterosis was 0.10 to $13.26 \%$ with a mean of $-6.09 \%$. Among the 16 hybrids, all exhibited the negative heterosis. Only two hybrids showed non-significant effect, another showed significant effect at $1 \%$ level. The heterosis of plant height ranged from -1.67 to $26.65 \%$ (Table 2). Among the 16 hybrids, 12 of them exhibited significant positive heterosis over the mid parent. Young and Virmani (1990) mentioned that heterosis for plant height did not change the plant type of hybrids from semi-dwarf to tall because the parents possessed semi dwarfing gene. Among the 16 hybrids, half (8) of them exhibited positive heterosis over the mid parent and half (8) of them had the negative heterosis for filled grains per panicle. Yolanda and Das (1996) observed considerable heterosis in the six quantitative traits especially in grains per panicle. Mishra and Pandey (1998) also reported that most of the higher yielding hybrids manifested positive heterosis for filled grains per panicle. The heterosis of yield per hill ranged from -2.86 to $128.4 \%$.

\section{Reciprocal effects}

In case of days to $50 \%$ flowering reciprocal effect was observed for all the crosses (Table 3). Only 4 crosses showed negative reciprocal effects but the cross BR-23 $\times$ FR-13A, FR-13A $\times$ PJM (st) and FR-13A $\times$ Arman sarder showed positive effects. Reciprocal effect for days to $100 \%$ flowering was observed for all the crosses studied. Among the crosses, six cross combinations (BR-23 $\times$ FR-13A, FR-13A $\times$ PJM (st), Chamara $\times$ FR-13A, Heachua $\times$ FR-13A, FR-13A $\times$ Khaiyamota and FR-13A $\times$ Arman Sarder) had positive effect while in other two crosses the effect was negative. This might be happened due to cytoplasmic maternal effect in either direction. Both negative and positive reciprocal effect was observed for days to maturity. The cross Heachua $\times$ FR$13 \mathrm{~A}$ and FR-13A $\times$ Khaiyamota showed negative reciprocal effects but the cross FR-13A $\times$ BR-22, BR-23 $\times$ FR-13A, FR-13A $\times$ PJM (st), PJT (st) $\times$ FR-13A, FR-13A $\times$ Arman Sarder and Chamara $\times$ FR-13A showed positive effects. Among the crosses, five pairs of combination had positive effect while in other three crosses the effect was negative for plant height. This might be happened due to cytoplasmic maternal effect in either direction. Reciprocal effect for panicle length was observed for all the crosses studied. Among the crosses, four pairs of combination had positive effect while in other four crosses the effect was negative. This might be happened due to cytoplasmic maternal effect in either direction. Both negative and positive reciprocal effect was observed for the trait number of primary branches per panicle. Most of the cross showed negative reciprocal effects. The crosses BR-23 $\times$ FR-13A, FR-13A $\times$ PJM (st), Chamara $\times$ FR-13A , Heachua $\times$ FR-13A and FR-13A $\times$ Arman Sarder showed negative reciprocal effects but the crosses FR-13A X BR-22, FR-13A $\times$ Khaiyamota and PJT (st) $\times$ FR-13A, showed positive effects. For number of tillers per plant, the cross FR-13A X BR-22, BR-23 $\times$ FR-13A, FR-13A $\times$ PJM (st), Heachua $\times$ FR-13A, FR-13A $\times$ Khaiyamota and FR-13A $\times$ Arman Sarder showed negative reciprocal effects but the cross PJT (st) $\times$ FR-13A and Chamara $\times$ FR-13A, showed positive effects. In case of yield per hill reciprocal effect was observed for all the crosses. All the crosses showed reciprocal effects. The crosses FR-13A $\times$ PJM (st), FR-13A $\times$ Khaiyamota and FR-13A $\times$ Arman Sarder showed negative reciprocal effects but the crosses FR-13A $\times$ BR-22, BR-23 $\times$ FR-13A, Heachua $\times$ FR$13 \mathrm{~A}, \mathrm{PJT}$ (st) $\times$ FR-13A and Chamara $\times$ FR-13A showed positive effects. Both negative and positive reciprocal effect was observed for the trait harvest index. All the crosses showed reciprocal effects. 
Table 2. Estimation of heterosis over mid parent in 16 crosses in T. Aman rice

\begin{tabular}{|c|c|c|c|c|c|c|c|c|c|c|c|c|c|c|c|}
\hline Hybrids & $\begin{array}{l}\text { Days to first } \\
\text { flowering }\end{array}$ & $\begin{array}{l}\text { Days to } \\
50 \% \\
\text { flowering }\end{array}$ & $\begin{array}{l}\text { Days to } \\
100 \% \\
\text { flowering }\end{array}$ & $\begin{array}{l}\text { Days to } \\
\text { maturity }\end{array}$ & $\begin{array}{l}\text { Plant } \\
\text { height } \\
(\mathrm{cm})\end{array}$ & $\begin{array}{l}\text { No. of } \\
\text { tillers per } \\
\text { hill }\end{array}$ & $\begin{array}{l}\text { Panicle } \\
\text { length } \\
(\mathrm{cm})\end{array}$ & $\begin{array}{l}\text { Primary } \\
\text { branches } \\
\text { per panicle }\end{array}$ & $\begin{array}{l}\text { Secondary } \\
\text { branches per } \\
\text { panicle }\end{array}$ & $\begin{array}{l}\text { Yield } \\
\text { per hill } \\
\text { (g) }\end{array}$ & $\begin{array}{l}\text { Yield per } \\
\text { tiller (g) }\end{array}$ & $\begin{array}{l}\text { Harvest } \\
\text { index }\end{array}$ & $\begin{array}{l}\text { Filled } \\
\text { grains per } \\
\text { panicle }\end{array}$ & $\begin{array}{l}\text { Spikelet } \\
\text { sterility } \\
(\%)\end{array}$ & $\begin{array}{l}\text { Wt.of } \\
100 \\
\text { grains (g) }\end{array}$ \\
\hline FR-13A $\times$ BR-22 & $-4.97 \mathrm{~ns}$ & $-7.8^{* *}$ & $-11.4 * *$ & $-9.76 \mathrm{~ns}$ & $18.7^{* * *}$ & $147^{* * *}$ & $14.2^{* * *}$ & $3.05 \mathrm{~ns}$ & $36.4 * *$ & $-3.73 * *$ & $-59.9 * *$ & $-56.0 * *$ & $-21.8^{* * *}$ & $235.6^{* *}$ & $10 . \mathrm{ns}$ \\
\hline FR-13A $\times$ BR- 23 & $-0.47 \mathrm{~ns}$ & $-3.6^{* *}$ & $-2.26 \mathrm{~ns}$ & $-1.48 * *$ & $19.3 * *$ & $73.0^{* * *}$ & $7.37 * *$ & $9.32 * *$ & $24.1 * *$ & $164.3^{* *}$ & $82.36 * *$ & $-41.1^{*}$ & $51.51^{* *}$ & $-2.87 \mathrm{~ns}$ & $-13 . n s$ \\
\hline FR-13A $\times$ PJM (st) & $-2.88 \mathrm{~ns}$ & $-5.06^{* *}$ & $-8.17 \mathrm{~ns}$ & $-8.43 * *$ & $3.02 \mathrm{~ns}$ & $5.31 \mathrm{~ns}$ & $-0.58 \mathrm{~ns}$ & $4.49 \mathrm{~ns}$ & $9.69^{*}$ & $33.51 * *$ & $27.31 * *$ & $618.3 * *$ & $19.85^{* *}$ & $6.9 \mathrm{~ns}$ & $19.35 \mathrm{~ns}$ \\
\hline FR-13A X PJT (st) & $-13.4 \mathrm{~ns}$ & $-10.8^{* *}$ & $-12.2^{*}$ & $-10.7^{* * *}$ & $31.6 * *$ & 156.1 ** & $10.0 \mathrm{~ns}$ & $14.28 * *$ & $4.30 \mathrm{~ns}$ & 219.0 ** & $30.82 * *$ & $-53.0 * *$ & $-26.5^{* *}$ & $149.2 \mathrm{~ns}$ & $-17 . \mathrm{ns}$ \\
\hline FR-13A $\times$ Chamara & $-2.38 \mathrm{~ns}$ & $-3.10^{* *}$ & $-8.29 \mathrm{~ns}$ & -1.89 ** & $-5.8 \mathrm{~ns}$ & $-20 \mathrm{~ns}$ & 3.03 ** & $-12.7^{* * *}$ & $-7.5 \mathrm{~ns}$ & $-6.84 * *$ & $19.58 \mathrm{~ns}$ & $41.93 \mathrm{~ns}$ & $1.39 \mathrm{~ns}$ & $8.79 \mathrm{~ns}$ & $14.28 \mathrm{~ns}$ \\
\hline FR-13A $\times$ Heachua & $-5.15 \mathrm{~ns}$ & $-0.80 \mathrm{~ns}$ & $-2.1 \mathrm{~ns}$ & $-5.14 * *$ & $11.8^{* *}$ & $106.4^{* * *}$ & $0.54 * *$ & $-16.5 \mathrm{~ns}$ & $4.27 \mathrm{~ns}$ & $128.4 * *$ & $-2.94 *$ & $79.16^{*}$ & $53.40 * *$ & $-57.57 * *$ & $59.18 \mathrm{~ns}$ \\
\hline FR-13A $\times$ Khaiyamota & $-10.71 \mathrm{~ns}$ & $-6.68 * *$ & $-13.7 *$ & $-8.95^{* *}$ & $24.0^{* *}$ & $96.96^{* *}$ & $15.9^{* *}$ & $-0.78 * *$ & $48.3 * *$ & $116.8^{* *}$ & $-14.8^{* *}$ & $-24 . *$ & $-20.3 * *$ & $40.6 \mathrm{~ns}$ & $-1.81 *$ \\
\hline FR-13A $\times$ Arman Sarder & $-13.5 \mathrm{~ns}$ & $-11.0^{* *}$ & $-14.9 \mathrm{~ns}$ & $-13.2^{* * *}$ & $26.5 * *$ & $85.94 * *$ & $7.81^{* * *}$ & $12.58 * *$ & $52.3^{* *}$ & $195.3^{* * *}$ & $107.1^{* *}$ & $558 . * *$ & $78.48 * *$ & $-27.7 \mathrm{~ns}$ & 28. ns \\
\hline BR-22 $\times$ FR-13A & $-7.2 \mathrm{~ns}$ & $-8.1^{* *}$ & $-11.7 \mathrm{~ns}$ & $-6.58 * *$ & $17.1^{*}$ & $135.9^{* *}$ & $10.4 * *$ & $6.09^{* *}$ & $41.6 * *$ & $2.86^{* *}$ & $-59.2^{* *}$ & $75.9^{* * *}$ & $-37.1 * *$ & $197.1^{* *}$ & $2.04 \mathrm{~ns}$ \\
\hline $\mathrm{BR}-23 \times \mathrm{FR}-13 \mathrm{~A}$ & $-1.11 \mathrm{~ns}$ & $-4.84 * *$ & $-9.03 *$ & $-4.01 * *$ & $27.9^{* *}$ & $123.0^{* * *}$ & $19.9^{* *}$ & $15.75^{* *}$ & 81.1 ** & $-27.9^{* *}$ & $-71.8^{*}$ & $90.69 * *$ & $38.57^{* *}$ & $146.79 * *$ & $-44.9^{*}$ \\
\hline $\operatorname{PJM}(\mathrm{st}) \times$ FR-13A & $-0.64 \mathrm{~ns}$ & $-2.30 * *$ & $-0.14 * *$ & $-0.10 \mathrm{~ns}$ & $-1.6 \mathrm{~ns}$ & $0.47 \mathrm{~ns}$ & $-5.07^{* *}$ & $-7.04 * *$ & $-28 . * *$ & $-5.73 * *$ & $11.31 *$ & $-7.09 \mathrm{~ns}$ & $-17.4 * *$ & $0.74 \mathrm{~ns}$ & $12.90^{*}$ \\
\hline $\mathrm{PJT}(\mathrm{st}) \times \mathrm{FR}-13 \mathrm{~A}$ & $-12.47 \mathrm{~ns}$ & $-11.4 * *$ & $-11.7^{*}$ & $-11.4 * *$ & $24.2 * *$ & $130.6 * *$ & $-1.38 \mathrm{~ns}$ & $-7.85 * *$ & $-6.5 \mathrm{~ns}$ & $142.9 * *$ & $21.01 *$ & $-69.3 * *$ & $-54.7 * *$ & $84.85 * *$ & $-17 . n s$ \\
\hline Chamara $\times$ FR-13A & $0.15 \mathrm{~ns}$ & $-9.00^{* *}$ & $-5.76 \mathrm{~ns}$ & $-1.47 * *$ & $3.79 \mathrm{~ns}$ & $92.5^{* *}$ & $5.32 * *$ & $-15.2 * *$ & $-6.68 \mathrm{~ns}$ & $94.04 * *$ & $10.16 \mathrm{~ns}$ & $12.90 \mathrm{~ns}$ & $-40.4 * *$ & $3.42 \mathrm{~ns}$ & $-3.5 \mathrm{~ns}$ \\
\hline Heachua $\times$ FR-13A & $2.16 \mathrm{~ns}$ & $-0.15 \mathrm{~ns}$ & $-2.14 \mathrm{~ns}$ & $-3.35^{* *}$ & $15.6 * *$ & $59.9 * *$ & $-1.28 \mathrm{~ns}$ & $-5.91 *$ & $1.068 \mathrm{~ns}$ & $56.60 * *$ & $-3.32 \mathrm{~ns}$ & $41.66 \mathrm{~ns}$ & $9.86^{*}$ & $-47.6 * *$ & 42. ns \\
\hline Khaiyamota $\times$ FR-13A & $-10.40 \mathrm{~ns}$ & $-10.1^{* *}$ & $-13.1 *$ & $-10.6^{* * *}$ & $12.2 * *$ & $69.6^{* *}$ & $22.47 * *$ & $7.09 *$ & $31.6 * *$ & $64.07 * *$ & $-16.5^{*}$ & $-13.3 \mathrm{~ns}$ & $1.00 \mathrm{~ns}$ & $45.08 \mathrm{~ns}$ & 45. ns \\
\hline Arman Sarder $\times$ FR-13A & $-7.8 \mathrm{~ns}$ & $-6.66 * *$ & $-12.9^{*}$ & $-7.15^{* *}$ & $13.4 * *$ & $17.8 \mathrm{~ns}$ & $0.76 \mathrm{~ns}$ & $-3.31 \mathrm{~ns}$ & $24.0^{* *}$ & $-23.2 * *$ & $-15.5^{*}$ & $61.63^{*}$ & $-47.0 * *$ & $76.30^{* *}$ & $-3.5 \mathrm{~ns}$ \\
\hline Mean heterosis & -5.69 & -6.35 & -8.75 & -6.09 & 15.13 & 80.12 & 6.85 & 0.20 & 19.38 & 71.92 & 4.09 & 82.23 & -0.71 & 53.73 & 8.34 \\
\hline No. of & 2 & 1 & 0 & 0 & 14 & 15 & 12 & 8 & 12 & 11 & 8 & 9 & 8 & 12 & 9 \\
\hline No. of negative heterosis & 14 & 15 & 16 & 16 & 2 & 1 & 4 & 8 & 4 & 5 & 8 & 7 & 8 & 4 & 7 \\
\hline
\end{tabular}

*indicates significant at $5 \%$ level and $* *$ indicates significant at $1 \%$ level 
Table 3. Reciprocal effect on eight crosses in T. Aman rice

\begin{tabular}{|c|c|c|c|c|c|c|c|c|c|c|c|c|c|c|c|c|}
\hline \multirow{2}{*}{$\begin{array}{l}\text { Parameter } \\
\text { Days to first flowering }\end{array}$} & \multirow{2}{*}{$\begin{array}{l}\text { FR-13A } \\
\times \text { BR-22 } \\
98.66\end{array}$} & \multirow{2}{*}{$\begin{array}{c}\text { BR-22 } \times \\
\text { FR-13A } \\
96.33\end{array}$} & \multicolumn{2}{|c|}{ Effects } & \multirow{2}{*}{$\begin{array}{c}\text { BR-23X } \\
\text { FR-13A } \\
103\end{array}$} & \multirow{2}{*}{$\begin{array}{c}\text { FR-13A } \times \\
\text { BR-23 } \\
103.66\end{array}$} & \multicolumn{2}{|c|}{ Effects } & \multirow{2}{*}{$\begin{array}{c}\begin{array}{c}\text { FR-13A } \times \\
\text { PJM (st) }\end{array} \\
101\end{array}$} & \multirow{2}{*}{$\begin{array}{c}\text { PJM (st) } \times \\
\text { FR-13A } \\
103.33\end{array}$} & \multicolumn{2}{|c|}{ Effects } & \multirow{2}{*}{$\begin{array}{c}\mathrm{PJT}(\mathrm{st}) \times \\
\text { FR-13A } \\
90\end{array}$} & \multirow{2}{*}{$\begin{array}{c}\text { FR-13A } \times \\
\text { PJT (st) }\end{array}$} & \multicolumn{2}{|c|}{ Effects } \\
\hline & & & -2.33 & $-\mathrm{ve}$ & & & 0.66 & $+\mathrm{ve}$ & & & 2.33 & $+\mathrm{ve}$ & & & -1 & $-\mathrm{ve}$ \\
\hline Days to $50 \%$ flowering & 100.33 & 100 & -0.33 & $-\mathrm{ve}$ & 104.66 & 106 & 1.3 & $+\mathrm{ve}$ & 103 & 106 & 3 & $+\mathrm{ve}$ & 94 & 94.66 & 0.66 & $+\mathrm{ve}$ \\
\hline Days to $100 \%$ flowering & 103 & 102.66 & -0.33 & $-\mathrm{ve}$ & 107.33 & 115.33 & 8 & $+\mathrm{ve}$ & 106.66 & 116 & 9.33 & $+\mathrm{ve}$ & 99.33 & 98.66 & -0.6 & $-\mathrm{ve}$ \\
\hline Days to maturity & 141.66 & 146.66 & 5 & $+\mathrm{ve}$ & 151.33 & 155.33 & 4 & $+\mathrm{ve}$ & 143 & 156.33 & 13.3 & $+\mathrm{ve}$ & 137 & 138 & 1 & $+\mathrm{ve}$ \\
\hline Plant height $(\mathrm{cm})$ & 130.06 & 128.26 & -1.8 & $-\mathrm{ve}$ & 141.73 & 132.13 & -9.6 & -ve & 127.13 & 121.33 & -5.8 & -ve & 142.26 & 150.73 & 8.46 & $+\mathrm{ve}$ \\
\hline No. of tillers per hill & 17.93 & 17.06 & -0.86 & $-\mathrm{ve}$ & 15.467 & 12 & -3.46 & -ve & 7.267 & 6.93 & -0.33 & $-\mathrm{ve}$ & 15.06 & 16.73 & 1.66 & $+\mathrm{ve}$ \\
\hline Panicle length $(\mathrm{cm})$ & 29.87 & 28.86 & -1.01 & $-\mathrm{ve}$ & 30.4 & 27.22 & -3.18 & -ve & 27.02 & 25.8 & -1.22 & $-\mathrm{ve}$ & 25.57 & 28.53 & 2.96 & $+\mathrm{ve}$ \\
\hline Primary branches per panicle & 11.26 & 11.6 & 0.33 & $+\mathrm{ve}$ & 12 & 11.33 & -0.66 & $-\mathrm{ve}$ & 10.86 & 9.66 & -1.2 & $-\mathrm{ve}$ & 8.6 & 10.66 & 2.06 & $+\mathrm{ve}$ \\
\hline Secondary branches per panicle & 31.8 & 33 & 1.2 & $+\mathrm{ve}$ & 41.533 & 28.46 & -13.0 & -ve & 27.53 & 18.06 & -9.46 & $-\mathrm{ve}$ & 19.53 & 21.8 & 2.26 & $+\mathrm{ve}$ \\
\hline Yield per hill (g) & 7.287 & 7.787 & 0.5 & $+\mathrm{ve}$ & 6.047 & 22.18 & 16.13 & $+\mathrm{ve}$ & 13.26 & 9.36 & -3.9 & $-\mathrm{ve}$ & 12.85 & 16.88 & 4.02 & $+\mathrm{ve}$ \\
\hline Yield per tiller (g) & 0.413 & 0.42 & 0.007 & $+\mathrm{ve}$ & 0.33 & 2.14 & 1.81 & $+\mathrm{ve}$ & 1.727 & 1.51 & -0.21 & $-\mathrm{ve}$ & 0.95 & 1.027 & 0.07 & $+\mathrm{ve}$ \\
\hline Harvest index & 0.17 & 0.68 & 0.51 & $+\mathrm{ve}$ & 0.82 & 0.253 & -0.56 & $-\mathrm{ve}$ & 5.567 & 0.72 & -4.84 & $-\mathrm{ve}$ & 0.15 & 0.23 & 0.08 & $+\mathrm{ve}$ \\
\hline Filled grains per panicle & 82 & 65.96 & -16.0 & $-\mathrm{ve}$ & 133.86 & 146.36 & 12.5 & $+\mathrm{ve}$ & 125.13 & 86.13 & -39 & $-\mathrm{ve}$ & 40.56 & 65.76 & 25.2 & $+\mathrm{ve}$ \\
\hline Spikelet sterility (\%) & 86.6 & 76.66 & -9.9 & $-\mathrm{ve}$ & 74.53 & 29.33 & -45.2 & $-\mathrm{ve}$ & 33.53 & 31.6 & -1.93 & $-\mathrm{ve}$ & 55.33 & 74.6 & 19.27 & $+\mathrm{ve}$ \\
\hline Wt. of 100 grains (g) & 2.7 & 2.5 & -0.2 & $-v e$ & 1.9 & 3 & 1.1 & $+\mathrm{ve}$ & 3.7 & 3.5 & -0.2 & & 2.4 & 2.4 & 0 & 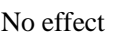 \\
\hline
\end{tabular}

Wh of 100 grains (g)

\begin{tabular}{|c|c|c|c|c|c|c|c|c|c|c|c|c|c|c|c|c|}
\hline \multirow{2}{*}{$\begin{array}{c}\text { Parameter } \\
\text { Days to first flowering }\end{array}$} & \multirow{2}{*}{$\begin{array}{c}\text { FR-13A } \times \\
\text { Chamara }\end{array}$} & \multirow{2}{*}{$\begin{array}{c}\text { Chamara } \times \\
\text { FR-13A }\end{array}$} & \multicolumn{2}{|c|}{ Effects } & \multirow{2}{*}{$\begin{array}{c}\begin{array}{c}\text { Heachua } \times \\
\text { FR-13A }\end{array} \\
102.33\end{array}$} & \multirow{2}{*}{$\begin{array}{r}\text { FR-13AX } \\
\text { Heachua } \\
95\end{array}$} & \multicolumn{2}{|c|}{ Effects } & \multirow{2}{*}{$\begin{array}{c}\text { FR-13A } \times \\
\text { Khaiyamota }\end{array}$} & \multirow{2}{*}{$\begin{array}{c}\begin{array}{c}\text { Khaiyamota } \\
\times \text { FR-13A }\end{array} \\
93.33\end{array}$} & \multicolumn{2}{|c|}{ Effects } & \multirow{2}{*}{$\begin{array}{c}\text { FR-13A } \times \\
\text { Arman Sarder } \\
91.33\end{array}$} & \multirow{2}{*}{$\begin{array}{c}\text { Arman Sarder } \\
\times \quad \text { FR-13A } \\
97.33\end{array}$} & \multicolumn{2}{|c|}{ Effects } \\
\hline & & & 2.66 & $+\mathrm{ve}$ & & & -7.33 & $-\mathrm{ve}$ & & & 0.33 & $+\mathrm{ve}$ & & & 6.0 & $+\mathrm{ve}$ \\
\hline Days to $50 \%$ flowering & 104 & 97.66 & -6.3 & $-\mathrm{ve}$ & 104 & 103.33 & -0.67 & -ve & 100 & 96.33 & -3.66 & $-v e$ & 95.66 & 100.33 & 4.66 & $+\mathrm{ve}$ \\
\hline Days to $100 \%$ flowering & 108.66 & 111.67 & 3 & $+\mathrm{ve}$ & 106.33 & 106.33 & 0 & 0 & 101.66 & 102.33 & 0.66 & $+\mathrm{ve}$ & 100.33 & 102.67 & & $+\mathrm{ve}$ \\
\hline Days to maturity & 55.33 & 156 & 0.66 & $+\mathrm{ve}$ & 154 & 141.333 & -12.6 & $-\mathrm{ve}$ & 144 & 14 & -2.66 & $-v e$ & 137.33 & 147 & 9.66 & $+\mathrm{ve}$ \\
\hline Plant height $(\mathrm{cm})$ & 115 & 126.73 & 11.7 & $+\mathrm{ve}$ & 141.13 & 136.47 & -4.66 & $-\mathrm{ve}$ & 156.2 & 141.26 & -14.9 & -ve & 160.66 & 144 & -16.6 & -ve \\
\hline No. of tillers per hill & 6.4 & 15.4 & 9 & $+\mathrm{ve}$ & 9.867 & 12.73 & 2.86 & $+\mathrm{ve}$ & 13 & 11.2 & -1.8 & $-\mathrm{ve}$ & 11. & 7.267 & -4.2 & -ve \\
\hline Panicle length (cm) & 26.46 & 27.07 & 0.58 & $+\mathrm{ve}$ & & 28.27 & 0.51 & $+\mathrm{ve}$ & 27.97 & 29.48 & 1.56 & $+\mathrm{ve}$ & & 27.47 & -1.92 & -ve \\
\hline Primary branches/ panicle & 9.133 & 8.867 & -0.26 & -ve & 10.07 & 8.933 & -1.13 & -ve & 8.4 & 9.06 & 0.66 & $+\mathrm{ve}$ & 11. & 9.733 & -1.6 & -ve \\
\hline condary brar & & & 0.2 & $+\mathrm{ve}$ & & 26 & 0.8 & $+\mathrm{ve}$ & 21.26 & 18 & -2.4 & $-v e$ & & 26 & -5.93 & -ve \\
\hline Yield per hill ( & 8.66 & 18.03 & 9.38 & $+\mathrm{ve}$ & & 15.02 & 4.72 & $+\mathrm{ve}$ & 14.40 & 10 & -3.50 & $-\mathrm{ve}$ & 20.027 & 5.20 & -14.8 & -ve \\
\hline Yield per tiller (g) & 1.44 & 1.33 & -0.11 & $-\mathrm{ve}$ & 1.03 & 1.037 & 0.001 & $+\mathrm{ve}$ & 0.857 & 0.84 & -0.01 & $-\mathrm{ve}$ & 2.11 & 0.86 & -1.25 & -ve \\
\hline Harvest index & 0.44 & & -0.09 & $-v e$ & 0.34 & 0.43 & 0.09 & $+\mathrm{ve}$ & 0.453 & 0.52 & 0.06 & $+\mathrm{ve}$ & 2.007 & 0.49 & -1.51 & -ve \\
\hline Filled grains per panicle & 111.26 & 65.33 & -45 & $-\mathrm{ve}$ & 115.06 & 160.67 & 45.6 & $+\mathrm{ve}$ & 64.97 & 82.33 & 17.3 & $+\mathrm{ve}$ & 193 & 57.27 & -135 & -ve \\
\hline Spikelet sterility(\%) & 24.33 & 23.13 & -1.2 & $-\mathrm{ve}$ & 29.93 & 24.27 & -5.6 & $-\mathrm{ve}$ & 27.66 & 28.53 & 0.86 & $+\mathrm{ve}$ & 20.73 & 50.6 & 29.8 & $+\mathrm{ve}$ \\
\hline Wt. of 100 grains $(\mathrm{g})$ & 3.2 & 2.7 & -0.5 & $-\mathrm{ve}$ & 3.5 & 3.9 & 0.4 & $+\mathrm{ve}$ & 2.7 & 4 & 1.3 & $+\mathrm{ve}$ & 3.6 & 2.7 & -0.9 & $-\mathrm{ve}$ \\
\hline
\end{tabular}


The crosses BR-23 $\times$ FR-13A, FR-13A $\times$ PJM (st), Chamara $\times$ FR-13A and FR-13A $\times$ Arman Sarder showed negative reciprocal effects but the crosses FR-13A $\times$ BR-22, Heachua $\times$ FR-13A, FR-13A $\times$ Khaiyamota and PJT $($ st $) \times$ FR-13A showed positive effects. Reciprocal effect was also effective for the trait grain per panicle. All the crosses showed reciprocal effects. Most of the crosses showed negative reciprocal effects. The crosses FR-13A $\times$ BR-22, BR-23 $\times$ FR-13A, FR-13A $\times$ PJM (st), Chamara $\times$ FR-13A and FR-13A $\times$ Arman sarder showed negative reciprocal effects but the crosses PJT (st) $\times$ FR-13A, Heachua $\times$ FR-13A and FR-13A $\times$ Khaiyamota showed positive effects. Reciprocal effect for yield per ha was observed for all the crosses studied. Among the crosses, four cross combinations had positive effect while other four crosses the effect was negative. This might be happened due to cytoplasmic maternal effect in either direction.

\section{REFERENCES}

Karim, Z., S. G. Hussain and M. Ahmed. 1990. Salinity problems and crop intensification in the coastal region of Bangladesh. BARC Soils Publication No. 33. $63 \mathrm{p}$.

Liang, G. H., C. R. Reddy and A. D. Dayton. 1971. Heterosis, inbreeding depression and heritability estimates in a systematic series of grain sorghum genotypes. Crop Sci. 12: 400-411.

Lokaprakash, R., G. Shivashankar, M. Mahadevappa, B. T. Shankare Gowda and R. S. Kulkarni. 1992. Heterosis in rice. Oryza. 29: 293-297.

Mishra, M. and M. P. Pandey. 1998. Heterosis breeding in rice for irrigated sub-humid tropic in north India. Oryza. 35(1): 8-14.

Patnaik, R. N., K. Pande, S. N. Ratho and P. J. Jachuck. 1990. Heterosis in rice hybrids. Euphytica. 49: 243-247.

Vaughan, D. 1980. Report of rice collection Trip in Faridpur, Pabna and Dinajpur Districts, Bangladesh. Bangladesh Rice Research Institute and International Rice Research Institute. 36p.

Yolanda, J. L. and L. D. V. Das. 1996. Studies on heterosis i hybrid rice. Oryza. 32:109110.

Young, J. and S. S. Virmani. 1990. Heterosis in rice over environments. Euphytica. 51: 87-93. 